\title{
SDN/NFV-enabled Satellite Communications Networks: Opportunities, Scenarios and Challenges
}

\author{
R. Ferrús ${ }^{1)}$, H. Koumaras ${ }^{2)}$, O. Sallent ${ }^{1)}$, G. Agapiou ${ }^{3)}$, T. Rasheed ${ }^{4)}$, M.-A. Kourtis ${ }^{2)}$, C. Boustie ${ }^{5)}$, P. \\ Gélard ${ }^{5)}$, T. Ahmed ${ }^{6)}$ \\ ${ }^{1)}$ Universitat Politècnica de Catalunya, Spain; ${ }^{2}$ National Centre for Scientific Research "Demokritos", \\ Greece; ${ }^{3)}$ Hellenic Telecommunications Organization S.A., Greece; ${ }^{4)}$ Center for Research and \\ Telecommunication Experimentation for Networked communities, Italy; ${ }^{5)}$ Centre National d'Etudes \\ Spatiales, France $;^{6)}$ CNRS-LaBRI, University of Bordeaux, France
}

This is the preprint version of the article published in Physical Communications, Elsevier:

R. Ferrus, H. Koumaras, O. Sallent, G. Agapiou, T. Rasheed, M.-A. Kourtis, C. Boustie, P. Gelard, T. Ahmed, SDN/NFV-enabled satellite communications networks: Opportunities, scenarios and challenges, Physical Communication (2015), http://dx.doi.org/10.1016/j.phycom.2015.10.007

(C2015. This manuscript version is made available under the CC-BY-NC-ND 4.0 license http://creativecommons.org/licenses/by-nc-nd/4.0/

\begin{abstract}
In the context of next generation 5G networks, the satellite industry is clearly committed to revisit and revamp the role of satellite communications. As major drivers in the evolution of (terrestrial) fixed and mobile networks, Software Defined Networking (SDN) and Network Function Virtualisation (NFV) technologies are also being positioned as central technology enablers towards improved and more flexible integration of satellite and terrestrial segments, providing satellite network further service innovation and business agility by advanced network resources management techniques. Through the analysis of scenarios and use cases, this paper provides a description of the benefits that SDN/NFV technologies can bring into satellite communications towards 5G. Three scenarios are presented and analysed to delineate different potential improvement areas pursued through the introduction of SDN/NFV technologies in the satellite ground segment domain. Within each scenario, a number of use cases are developed to gain further insight into specific capabilities and to identify the technical challenges stemming from them.
\end{abstract}

\section{INTRODUCTION}

The combination of satellite and terrestrial components to form a single/integrated telecom network has been regarded for long time as a promising approach to significantly improve the delivery of communications services [1][2]. Despite the important and continued advances in satellite communications technologies, satellite communication offerings have not evolved at the same pace as terrestrial communications systems have done due to much lower economies of scale and inherent associated technological complexities [3]. In this context, the satellite industry is clearly committed to revisit and revamp the role of satellite communications in the path towards next generation 5G networks [4][5]. Indeed, considering the actual and future challenges being pursued under $5 \mathrm{G}$, it is of utmost importance that next 
generation network architecture supports multiple layers and heterogeneity of network technologies, including satellite communications.

Advances in satellite communications are being addressed from multiple angles such as High Throughput Satellites (HTS) with multi-beam and frequency reuse capabilities [6], lowcost micro-satellites in low-orbit constellations and higher millimetre wave frequencies [7][8] and more flexible payloads components to dynamically modify satellite antenna beam patterns in orbit to respond to market demands [9]. Likewise, within the satellite ground segment (satellite hubs, satellite terminals and networking equipment within the satellite networks), main innovations are being pursued through the introduction of Software Defined Networking (SDN) and Network Function Virtualisation (NFV) technologies [10][11]. The handicap here is that, in current satellite ground segment network architectures (referred simply to as satellite networks in the following), there is a lack of prevalent standards and much functionality is mainly deployed on vendor-specific network appliances, which execute specific functions. This leads to a network infrastructure settings too prone to vendor locking, too complicated to manage when operating together solutions from various vendors, and too hard to change in behaviour. With the introduction of SDN and NFV, greater flexibility is expected to be achieved by satellite network operators, in addition to the much-anticipated reduction of both operational and capital expenses in deploying and managing SDN/NFVcompatible networking equipment within the satellite networks. Furthermore, the adoption of SDN/NFV into the satellite networks is seen as a key enabler towards more flexible and agile integration of satellite and terrestrial networks. This should allow overcoming several existing limitations in terms of operational flexibility, evolvability and end-to-end service provisioning.

Through the description of relevant scenarios and use cases, this paper investigates how SDN and NFV technologies can enhance the operation of the satellite networks and the deployment and management of communications services across hybrid satellite-terrestrial configuration variants. Though scenarios and use cases can be approached from multiple perspectives (business-centric, service-centric, technology-centric, network architecturecentric, actor-centric, etc.), the selected scenarios presented in this paper have been defined to delineate different potential improvement areas pursued through the introduction of SDN/NFV technologies in the satellite domain:

- Scenario 1 - Virtualisation and multi-tenancy of satellite networks. This scenario focuses on the exploitation of SDN/NFV technologies internally, within the satellite ground segment. In particular, the scenario aims to improve flexibility and reconfigurability in the delivery of satellite network services by supporting virtualisation (i.e. softwarisation) of key satellite network functions together with network abstraction and resource control programmability. In addition, enhanced multi-tenancy support is envisioned to facilitate the sharing of satellite network infrastructures and the implementation of satellite virtual network operator (SVNO) models.

- Scenario 2 - 4G/5G satellite backhauling services. Building on the capabilities envisioned under Scenario 1 for SDN/NFV-enhanced satellite networks, the improvement area pursued here is the combination of satellite and terrestrial networks when the satellite part is used for the delivery of backhauling services. In particular, this scenario focuses on the use of satellite communications so as to provide connectivity to ground-based network components, which may be either fixed platforms, e.g. fixed base stations, or moving ones, e.g. small cells installed in moving vehicles such as ships or trains. 
- Scenario 3 - Satellite-terrestrial hybrid access services. Building on the capabilities envisioned under Scenario 1 for SDN/NFV-enhanced satellite networks, the improvement area pursued here is the delivery of satellite-terrestrial hybrid access services. Hybrid access is used in this paper to refer to access networks combining a satellite component and a terrestrial component in parallel [12]. In particular, Scenario 3 focuses on the cost-efficient integrated operation of satellite communication services as complementary to terrestrial fixed and mobile access so as to increase the Quality of Service / Quality of Experience (QoS / QoE) level delivered to endusers.

For each scenario, a number of use cases are provided to shed light on specific capabilities or features and to identify key technical challenges lying ahead on the way towards the envisioned SDN/NFV-enabled satellite-terrestrial networks.

The rest of the paper is organised as follows. Section 2 provides some background material on current satellite network architectures and SDN/NFV technologies. Then, Section 3 to Section 5 are devoted to analyse each of the identified scenarios. Finally, main conclusions are drawn in Section 6.

\section{BACKGROUND}

Figure 1 illustrates a general reference model for a multi-gateway satellite ground segment for satellite broadband communications [13][14]. It is structured in three main subsystems:

- The access subsystem, commonly referred to as the satellite access network. This includes the satellite gateways (GWs) and the satellite terminals (STs), which are interconnected through the resource of one or several channels (transponders) of a communication satellite. It can use a variety of network topologies (star, multi-star, mesh or hybrid star/mesh) and provide a variety of types of L2 and L3 connectivity with bidirectional links. A GW can provide access to different logically separated groups of STs.

- The core subsystem, commonly referred to as the satellite core network. This is an aggregation network that interconnects different satellite GWs located in the same or different teleport as well as the network nodes located in some Points of Presence (PoPs) to interconnect with other operators, corporations and Internet Service Providers (ISPs). Typically, the satellite core network is built around an optical backbone with switching and routing equipment nodes based on IP/Multi-Protocol Label Switching (MPLS) and/or Carrier Grade Ethernet technologies.

- The control and management subsystems, composed of network elements such as the Network Control Centre (NCC) and the Network Management Centre (NMC), the former providing real-time control of the satellite network (e.g. connection control including the signalling necessary to set up, supervise and release connections) and the latter in charge of the management of the system elements of the satellite network (e.g. configuration, fault, performance, accounting and security management).

A satellite GW typically comprises: the Outdoor Unit (ODU), composed of the antenna and radio head units; the Baseband subsystems, composed of the Forwarding Link (FL) subsystem and the Returning Link (RL) subsystem for satellite access and transmission; and a set of Network Functions, including Performance Enhancing Proxy (PEP) functions (e.g. 
TCP optimization, HTTP and content caching) to improve the higher layer protocols performance and L2/L3 switching and routing functions and interfaces for the interconnection with the satellite core network (e.g. IP router, Ethernet switch, IP/MPLS router). Functionally, the satellite GW is in charge of executing user plane functions, under the control and management of a NCC/NMC. Typically, the GW and its associated NCC/NMC are co-located and commonly referred to as satellite hub (i.e. GW/NCC/NMC). With regard to STs, the group of ST that form part of the same satellite network are controlled by a unique NCC. STS that deliver satellite broadband access are typically equipped with an IP router and/or an Ethernet switch to interwork with any attached external end-user equipment. The network equipment on the user side (e.g. routers, switches, firewalls, etc.) used to connect the enduser hosts to the ST is collectively referred to as the Customer Premises Equipment (CPE). It's worth noting that this satellite network architecture is compliant with the more general reference architecture promoted by the Broadband Forum [15] to build a converged broadband multi-service network to support emerging as well as existing services.

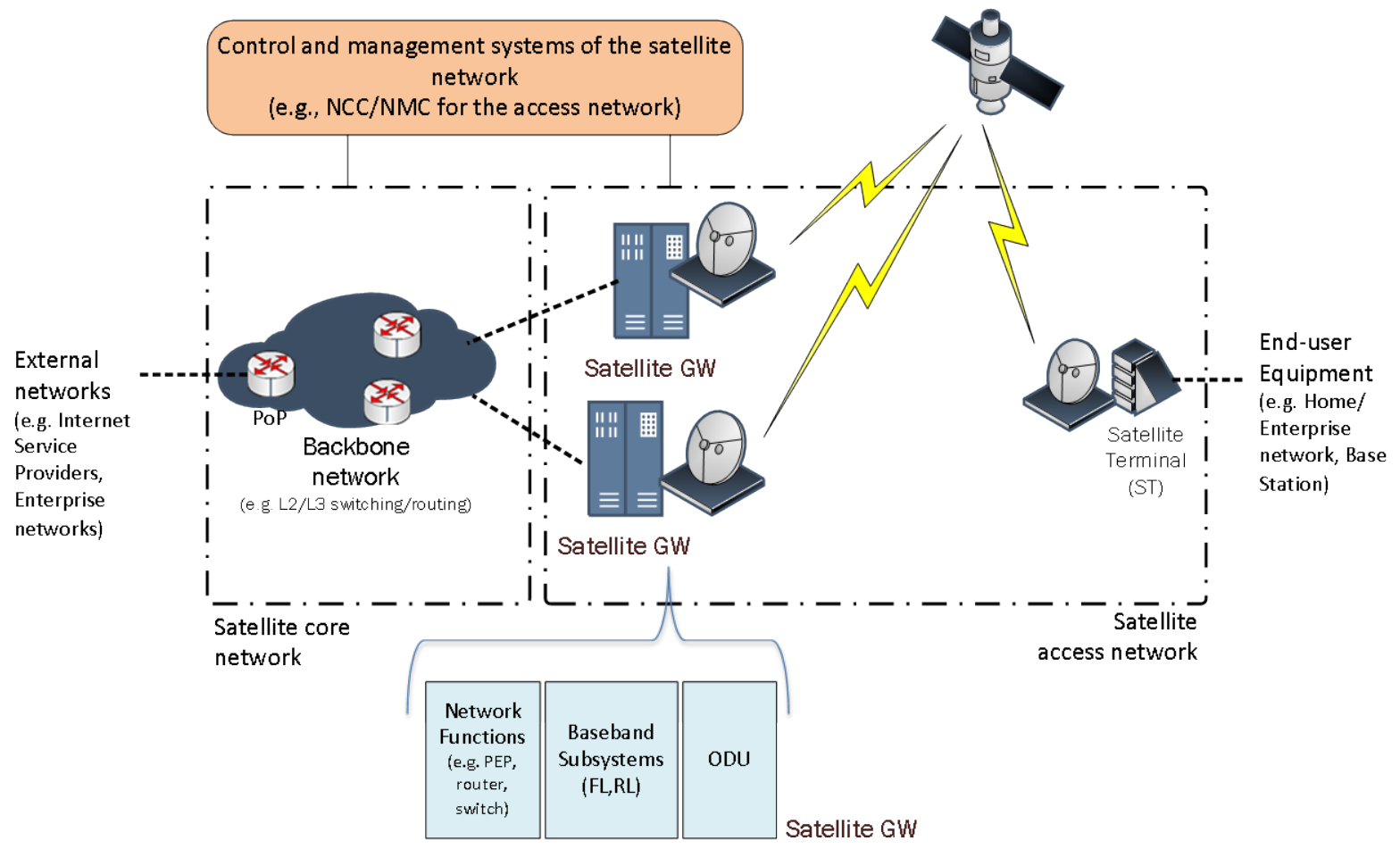

Figure 1. Reference architecture for a typical multi-gateway satellite network

The operation and delivery of satellite broadband communication services involve mainly the following roles [16]:

- Satellite Operator (SO), which is responsible for maintaining, managing, deploying and operating the satellite platform. The SO business involves launching and operating satellites and selling their transponder capacity to Satellite Network Operators (SNOs).

- Satellite Network Operator (SNO), which owns and is responsible for maintaining, managing, deploying and operating the satellite network infrastructure, i.e. leasing satellite transponders and providing the associated ground segment equipment (e.g. teleport and hub infrastructure). It is responsible for the centralized management and control functions in infrastructures supporting multiple satellite networks, each one controlled by its own NCC. It offers a given coverage, connectivity and bandwidth to 
Network Access Providers (NAPs), managing the partitioning of the resources between the NAPs and deciding on the satellite payload configuration.

- Network Access Provider (NAP), which uses the services from one or more SNOs to provide bulk transmission resources to the Service Providers (SPS). The NAP is responsible of managing and operating the network access elements including STs and one or more satellite GWs. A given GW/NCC is under the responsibility of only one NAP. The NAP typically shares its network capacity between several SPs.

- Service Provider (SP), which sells the service and/or the equipment to customers (end-users or other service providers). The SP is responsible of managing and operating the related service provider elements in the STs and in one or more satellite GWs (the SPs are responsible for the network and above layers of the ST and $\mathrm{GW})$. The SP gives access to a wide range of services involving terrestrial networks or not. Several types of SP can be identified. They can be Network Service Providers (NSP) or Application Service Providers (ASP). Examples of NSP are Internet Service Providers (ISP) or Corporations (e.g. VPN). Examples of ASP are Multicast Service Provider (MSP) and Internet Telephony Service Provider (ITSP).

Some of the above roles can be played separately or jointly by the same or different business actors (e.g. a company performing the role of SNO and NAP). While there are many particular cases, the main traditional and emerging business models and actors with the continued increase of HTS capacity [17] are illustrated in Figure 2:

- Vertically Integrated Model. In this model, a single company, referred to as SNO, plays the roles SO, SNO, NAP and SP. Therefore, a single actor owns and operates the entire value chain: the satellite, teleport, hub infrastructure, network operations, service provision and customer relationships.

- Managed Service Model. In this model, there is also a SNO company that owns and operates the satellite network infrastructure and network operations (i.e. plays the SO, SNO and NAP roles) but now it relies on other companies to resell satellite communications services and manage customer relationships. These companies play a pure SP role and are commonly referred to as SP or distributors. In a variant of this model, known as the partially managed model, the SP company is also enabled to have certain control over the network operations and service provisioning, so that some degree of customisation by the SP of the offered satellite services on top of the hosting SNO's networks is possible.

- Satellite Virtual Network Operator (SVNO) Model. In this model, a company focused on service delivery can get leased some bandwidth with partial satellite hub equipment control and management from a hosting SNO, achieving full control of its own satellite network and customers (i.e. the SVNO company plays the SP and NAP roles). In a variant of this model, known as Hub Co-Location Model or full SVNO, the company focused on service delivery co-locates its own satellite hub equipment within the teleport of the hosting SNO (i.e. the full SVNO now plays the SP, NAP and a partial SNO role). 


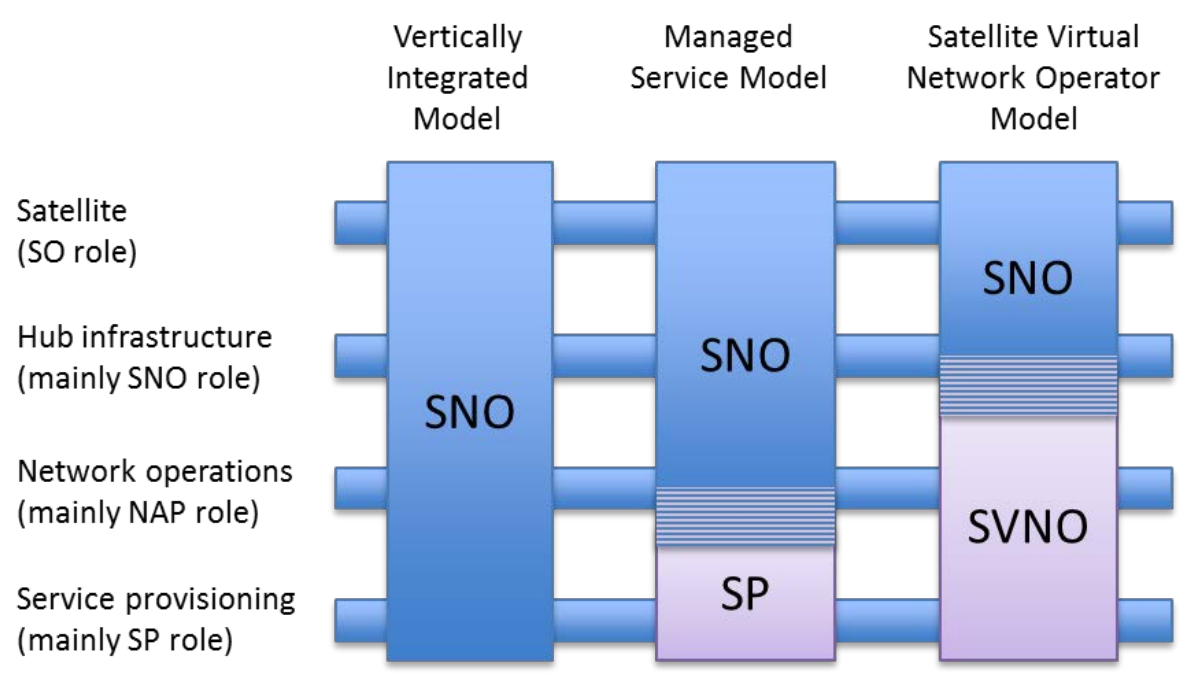

Figure 2. Business models and actors in broadband satellite communications

Given that terminology used for roles and actors shows some overlapping, unless explicitly mentioned otherwise, the terms SNO, SP and SVNO are used in the following to refer to the actors mentioned in the previous business models and illustrated in Figure 2. References to specific roles are only introduced if necessary for the sake of clarity.

Some of the key limitations of current satellite networks under the focus of the satellite industry are the following:

- Establishment and configuration of networking services across satellite and terrestrial segments is mostly performed manually, using static and complex configuration and thus involving considerable setup and reconfiguration delays, as well as high associated operating and maintenance costs.

- New network technologies, algorithms and protocols cannot be rapidly introduced into the market since they involve time-consuming and costly satellite communications and terrestrial hardware upgrades and are thus associated with significant CAPEX investments.

- Lack of flexibility in configuring, provisioning and managing of the satellite resources to achieve a better match with users' demand and to optimize the resources in use. Modifications in the configuration of satellite resources for one user are especially critical since they may impact on other users. Time consuming and costly tasks are needed for testing and validation when there is a change in the frequency arrangements (even though it's possible to manage a number of validated frequency arrangements).

- Satellite communication services are mostly associated with plain connectivity (e.g. L2/L3 connectivity with or without QoS), with limited ability to insert on-demand innetwork services (e.g. firewalling, proxying for traffic optimization, caching, media transcoding, etc.) for network-side and edge traffic processing.

- Many satellite specific settings and the lack of common prevalent standards for the integration with terrestrial systems do not provide a transparent manner for the applicability and continuity of policies for e.g. routing, QoS, security, management and connectivity (Ethernet, MPLS, etc.), across both segments.

- Limited control by SPs for global resource management when relying on multiple network providers for the delivery of satellite communications services. The purpose of a SP is to be able to offer its entire catalogue of services to its customers through 
different satellite networks (likely operated by different SNOs), in a transparent manner. However, the interconnection with and management of various and diverse sets of equipment, with specific characteristics in each satellite network, raise too much complexity.

In the terrestrial domain, limitations such as the lack of automation, limited flexibility in scaling/upgrading networking equipment and services noted above are also present but gradually being confronted via a major technological transition sustained in the still emerging concepts and technologies related to SDN and NFV. On the one hand, SDN [18] decouples the control and user planes of the networking equipment and logically centralizes the network intelligence (i.e. control plane), while the underlying network infrastructure (i.e. user plane) is abstracted for external applications requesting services through that control plane. In this sense, SDN constitutes a new architectural paradigm to design network functions (e.g. routing, load balancing, firewalling, NAT) that provides abstraction, facilitates programmability, and simplifies management functionalities through standard interfaces. On the other hand, the scope of NFV [19] is to decouple network functions from proprietary hardware, making it possible to run such functions in general-purpose commodity servers, switches, and storage units, which could be deployed in a network operator's data centre. By implementing network functions in software (i.e. Virtual Network Functions, VNFs) that can run on a range of industry-standard servers, operators can be freed from the need to install new proprietary equipment within the network itself. Furthermore, NFV provides improvement in the utilization of physical resources by allowing multiple instances of the same or different VNFs to coexist over a common pool of compute, network and storage resources. A management layer then orchestrates the resources to support VNFs as needed, in a fluid, dynamic, and real-time environment. Unlike SDN, NFV does not necessarily introduce any architectural change into the network functions (e.g. a firewall function can be the same irrespective of whether it is provided as a hardware appliance or as a software package - a VNF - to run on a cloud environment). In turn, NFV is more related to how a given network function is provisioned (e.g. as a hardware appliance or as a VNF to be executed in a NFV Infrastructure Point-of-Presence [NFVI PoP]). From this perspective, the main expected benefits with NFV are lower OPEX, greater flexibility (i.e. easily scaling network resources up and down), and easier network management. It's worth noting that a NFV infrastructure may rely on underlying SDN-enabled network functions to realise the connectivity between VNFS (and also between internal components of the same VNF) deployed in one or across several NFVI PoP(s).

\section{Virtualisation and Multi-Tenancy OF SATELLITE NETWORKS}

In line with the current trend regarding infrastructure and network sharing in mobile communications, SNOs are moving toward opening their infrastructure to be shared by multiple tenants. This can be seen as offering wholesale access to network resources (bandwidth) up to further offering a high controlling level on network equipment and management of high and low level functionalities (e.g. configuring the time/frequency plan for satellite access) to the extent that the SNO's network infrastructure can be partitioned into logically isolated virtual satellite networks (i.e. network slicing). This turns network slicing into a functionality of prime importance for satellite networking. With network slicing, SNO can selectively expose resources and operations in their hosting network to different end-users 
(e.g. government, private enterprise), SPs/distributors and other sub-tier service providers such as SVNOs.

The management of the lifecycle of network slices in terms of adding a new network service, increasing its capacity and managing its state information can be very challenging within current satellite networks since it requires the reconfiguration of multiple hardware and software components including the satellite gateway, the routers, switches and associated application servers to support its integration and management operations. These operations can be very complex and may lead to inconsistent configurations. The only way it was considered up-today to provide consistency is to have network services manually deployed, massively overprovisioned, and statically managed using a pre-determined Service Level Agreement (SLA). Usually, this leads to inflexible management operations, non-optimized network usage, and low performance.

With the advent of SDN/NFV enabling technologies, the network slicing process is highly facilitated. When compared to traditional network virtualisation via tunnels and encapsulation, SDN-controlled virtualisation can achieve much higher resource efficiency, due to its higher dynamicity and granularity to manage traffic flows, and also much faster service setup times. State-of-the-art SDN controllers can act as the necessary virtualisation middleware to enable the abstraction of network slices as well as their use by applications on multi-tenant basis via appropriate Northbound Interfaces (NBIs) [18]. In addition to SDN-controlled network virtualisation, a SNO can deploy specific Virtual Network Functions (VNFs) for a particular tenant or a set of tenants such as virtual PEP (VPEP), virtual firewall, virtual bandwidth capacity and traffic optimizer (e.g. virtual Load Balancer), and virtual traffic analyser including data collection and deep packet inspection. This allows the efficient sharing of satellite network capacities in flexible and multi-tenant manner while providing support of automate batch control, interaction with the network elements at a higher level, and enabling rapid services provisioning and deployment. It also allows for a more flexibly reconfigurable network that can accommodate multiple tenants requesting different isolated capabilities. In the end, the aim is to satisfy the requirements of every envisioned service having different and diverse requirements (e.g. high-speed, large-volume, low-latency network connectivity for voice, video, database communications). Therefore, the introduction of SDN/NFV within the satellite networks is expected to contribute toward the achievement of the following objectives:

- To optimize and to share efficiently the satellite infrastructure and networks resources.

- To offer wide range of services such as bandwidth on demand, QoS on demand, and resource sharing or aggregation in order to improve the profitability of resources available and customer satisfaction.

- To allow the use of the satellite network as a multiservice network that offers the support of a wide range of services and applications, each of them potentially requiring specific performance guarantees.

- To allow for more dynamic and efficient sharing of the satellite core network infrastructure by several SNOs as well as by other players (e.g. SP, SVNOs).

- To simplify integration and management of network services (such as firewall, loadbalancer, QoS optimizer, etc.) by providing interface for resources provisioning and invocation. 
- To improve the hybridization of terrestrial and satellite network infrastructure through the establishment of common and shared interfaces for resource management, orchestrator, and control.

- To offer support of SVNO capabilities in terms of isolated resources, dedicated or shared network management system, ability to monitor and control individual resources usage and to provide predictable performance.

- To allow resources adjustment when SVNO expand or shrink their virtual resources and to provide efficient virtual to physical resources mapping.

- To support agile deployment of new services that keep pace with the rapid growth and change of tenants' demands.

In the framework of these objectives, three use cases are addressed in the following subsections to gain more insight into a number of key relevant features within this scenario and identify key technical challenges lying ahead.

\subsection{SDN-based satellite bandwidth on demand}

The aim of the SDN-based flexible satellite bandwidth on demand is to advance the typical satellite broadband access service with the customer to be able to dynamically request and acquire bandwidth and QoS in a flexible manner. This is a first step toward introducing more agility and dynamicity in managing network resources, including Radio Resource Management (RRM) of the satellite links. Agility means that the satellite network configuration, dimensioning and adjustment can be performed in real-time (or near real-time) to better fulfil the customer's expectation but also to optimize the utilization of network resources.

Nowadays, many SNO's customers such as ISP, data-centre service provider, cloud provider, Content Delivery Network (CDN) provider or end-users (e.g. private enterprises) may need transient particular network resources for its Wide Area Network (WAN) connectivity needs during a specific time period and between particular network locations. These transient resources can be used for backup but also to absorb some large demand on the WAN connections or to manage extra demand for sites interconnection (inter-enterprise collaboration project, big-data file sets downloading, etc.). All of these trends result in bandwidth demand peaks that can be 10 to 20 times greater than their mean, with the peaks lasting anywhere from less than an hour to several weeks or more [20][21]. This procedure is referred to as on-demand bandwidth and can be generalized to on-demand network service including on-demand QoS and on-demand service class aiming to change dynamically and on the fly the configuration of the different QoS profiles and services classes. 


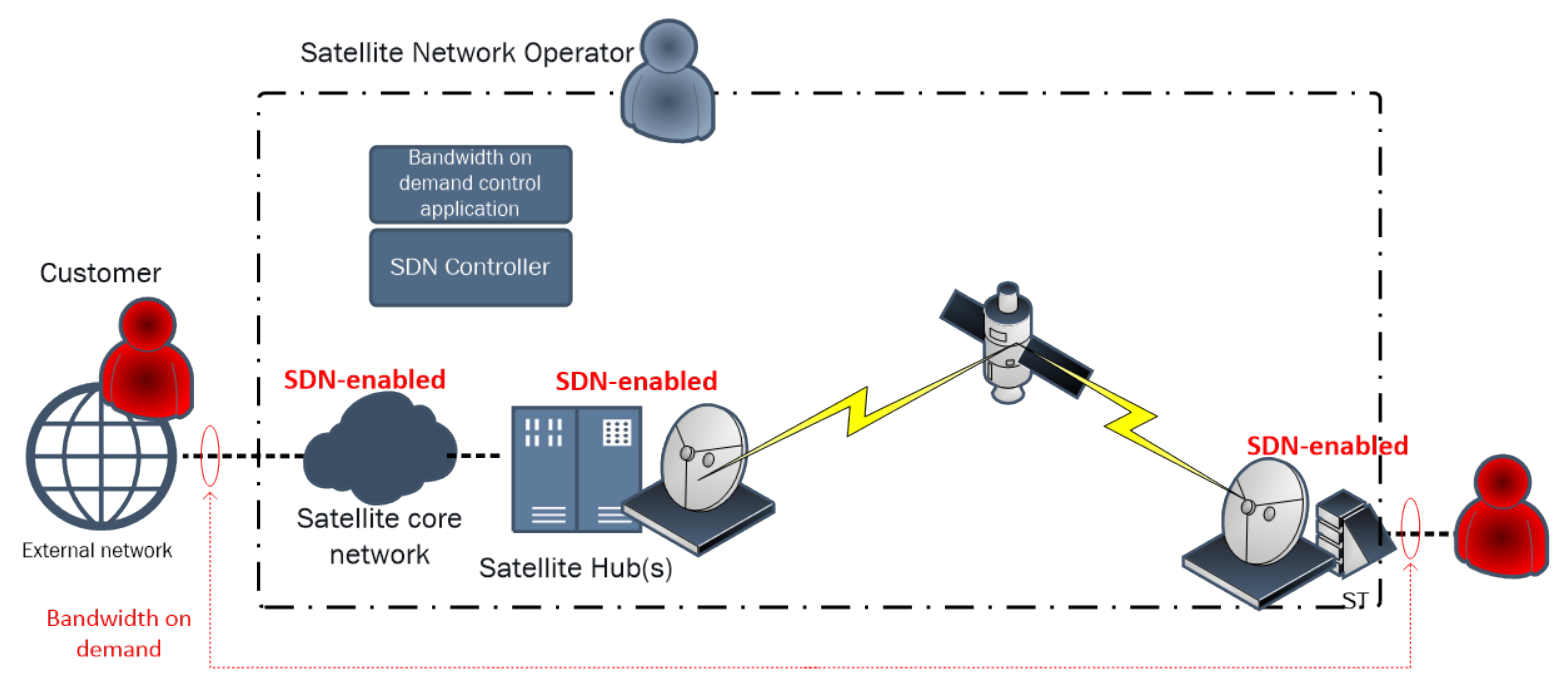

Figure 3. Illustration of Use Case "SDN-based satellite bandwidth on demand"

Enabling users to invoke elastic network resources when needed leads to higher application performance and business efficiency. The elastic network resource provision requires that traffic control, inspection, prioritization and also metering capabilities are present across the satellite network components. Although these operations can be achieved via traditional networking technologies, their implementation via SDN offers much more flexibility and agility. Among the advances of applying flexible on demand bandwidth supported by a SDN architecture with a programmatic northbound API, are the centralized and granular controls over the networking infrastructure, enabling customers to automatically request dynamic changes to bandwidth allocation and other QoS parameters, either immediately or scheduled in the future. Furthermore, a centralised SDN architecture can leverage per-flow management to cost-effectively provide guaranteed performance on a per-connection or flow basis to meet SLA requirements. Therefore, SDN-driven traffic engineering is feasible and quite effective to manage at the ground segment, where capacity among services and customers can be easily repartitioned.

As illustrated in Figure 3, SDN capabilities are anticipated across the satellite network, including the satellite hubs and terminals. These SDN capabilities will be managed by a centralized SDN controller with a global view of the satellite network resources, including data plane resources within the satellite core network as well as proper abstractions of the resources of satellite hubs to control the offered bandwidth in the access network. In the satellite core network, including the network functions within the satellite GW, the SDN controller will dictate the appropriate flow rules, configuring the precedence and the policing as necessary. However, the case of providing flexible and on demand bandwidth in the satellite access subsystem is more challenging due to resources constraints and their statistical multiplexing. It is worth noting that the connectivity of the satellite access network is divided into two distinct parts: forwarding link (FL) and returning link (RL). The FL can be based on a Time Division Multiplexed (TDM) broadcast downstream channel from a central hub location and shared by a number of remote sites. On the other hand, in the RL each remote transmits to the hub using techniques such as Demand Assigned Multiple Access (DAMA) or dedicated Single Channel Per Carrier (SCPC). DAMA techniques allow achieving statistical multiplexing while providing satellite terminals with committed information rates (CIR). Therefore, especially for the $\mathrm{RL}$, the approach of elastic provision is not so straightforward. The reason is that per-customer $\mathrm{RL}$ throughput also depends on the radio 
resources assigned. Therefore, in order to provide further bandwidth adaptation flexibility in the satellite access subsystem, it is necessary to couple SDN control with the RRM functions of the satellite hub (RRM covers interface management, power control, fading mitigation, handover, admission control, congestion control, bandwidth allocation, and packet scheduling). In this case, a vertical cross-layer integrated management architecture [22] could be considered as most relevant since various OSI layers information (L1 up to L7) can be integrated in the management and control.

Key technical challenges arising in this use case are:

- How SDN techniques can be applied and supported by satellite gateways and remote terminals to meet the requirements of this use case.

- How to efficiently provide transient on-demand network service without affecting normal operation of other users.

- How to perform fast provisioning of satellite network resources and to perform dynamic network configuration to meet the demand.

\subsection{Satellite virtual network operator}

The SVNO model considers that a part of satellite network resources and services of the hosting network are shared with third party operators while offering a high controlling level on network equipment and management functionalities. A SVNO does not have its own licensed frequency allocation of radio spectrum, nor does it necessarily have the entire infrastructure required to provide the service. The SVNO model allows new players to enter quickly a market and to develop niche opportunities without huge investments. Conceptually, a SVNO is a customer of the hosting satellite network providers. The design of the SVNO model has largely been based on a hardware option which aims to create a separate network on top of the hosting network operator using dedicated hardware and software tools. The advantages for SVNO are many. First, SVNO operates his own network with low cost and low investment since no complex hardware and software such as teleport and satellite hub infrastructure are needed to be maintained. Second, SVNO benefits from flexible and changing resources upgrade according to their needs while elasticity is possible by adjusting SLA according to customer dynamic demands.

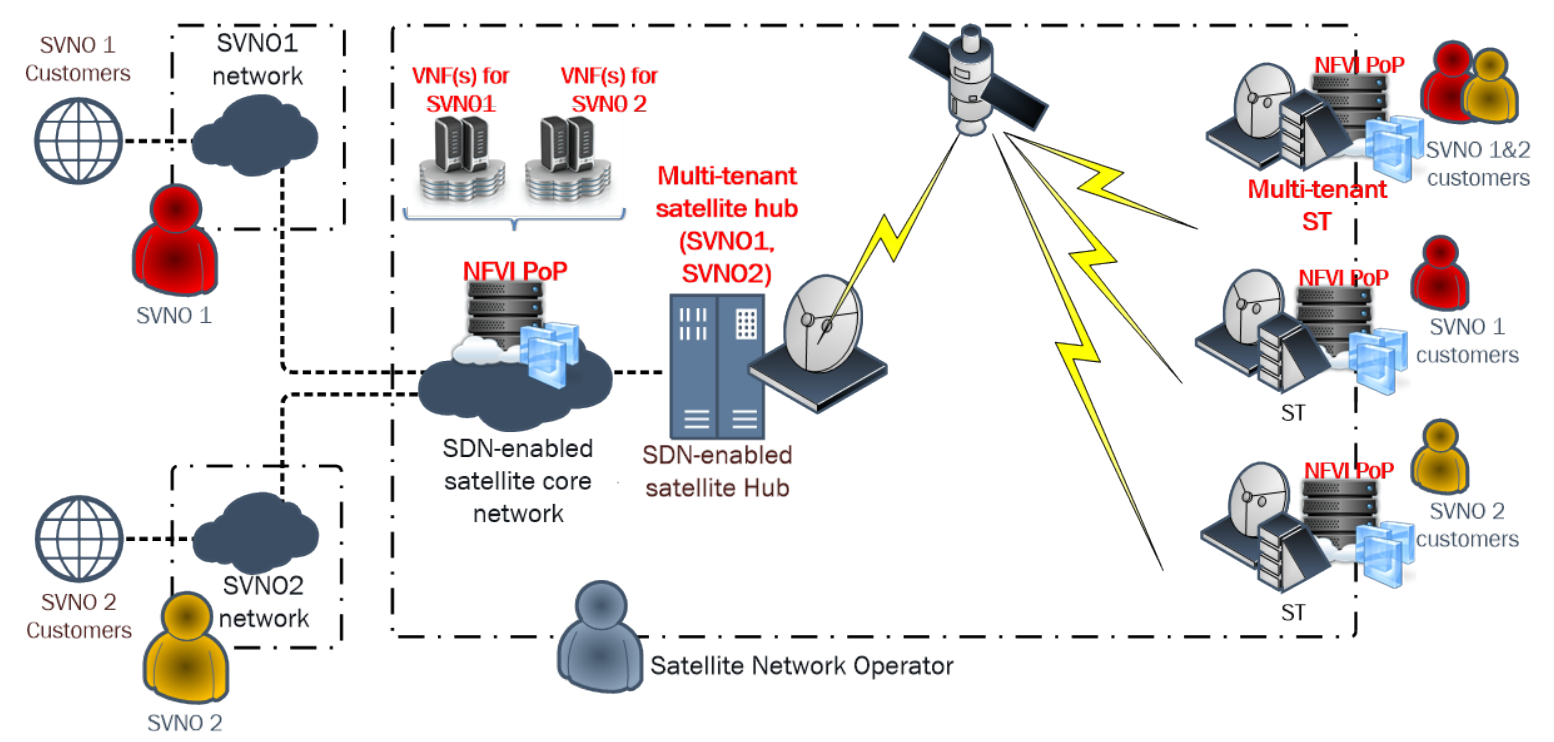




\section{Figure 4. Illustration of Use Case "Satellite virtual network operator"}

In this context, the aim of this use case (illustrated in Figure 4) is to research how the SDN and NFV techniques can facilitate the creation of a virtual network by abstracting the underlying network resources so that the hosting network is "sliced" for multi-tenants (i.e. different SVNOS) and then presented as a dedicated portion of the network resources for achieving service isolation and offering seamless communications services. To achieve such a goal, SDN is envisioned to be used to i) reserve SVNO capacity and ii) establish dedicated SVNO traffic flows within the satellite network. On the other hand, NFV will be applied to satisfy the needs that each tenant exposes in terms of some required network services such as firewalling, NAT, PEP, etc., which are advisable to be present before the satellite access subsystem, for the sake of saving satellite capacity. Such capabilities cannot be currently provided per SVNO in a flexible manner since it usually implies the deployment and integration of new hardware appliances within the hosting network. The NFV and SDN techniques promise to alleviate these limitations by allowing network functions in the form of VNFs to be acquired on demand by the SVNO and instantiated either at the ST (e.g. in a light NFVI PoP co-located with the ST) or in one or a number of central shared NFVI PoPs within the satellite network. Some functions, such as PEP and application classification, could be installed at both ends. Also multi-tenancy at STs is envisioned so that multiple SVNOs can be attached to the same ST but still have customised network services. Key technical challenges arisen in this use case are:

- How to enhance multi-tenancy and sharing support of the satellite hub components (GW/NCC/NMC) among multiple SVNOs through SDN/NFV techniques, allowing each SVNO to gain advanced control, more flexibility, and programmability of its allocated resources within the satellite hub.

- How to integrate and manage VNFs per SVNO in a multi-tenant satellite network.

- How to isolate the required SVNO capacity from other SVNOs and from the hosting SNO and guarantee individual managed resources and predictable performance with appropriate SDN policy techniques.

- How to provide resources adjustment when SVNO expand or shrink their resources, providing monitoring and control of individual SVNO resources, and finally providing flexible and efficient virtual to physical resources mapping.

- How to support of agile deployment of new network services and new VNFs that keep pace with the rapid growth and change of customer demands.

\subsection{Satellite Network as a Service (SatNaaS)}

By exploiting the virtualisation paradigm, the use-case described herein extends the SVNO concept from the plain slicing of satellite network capacity with NFV support for some customised network services toward the full virtualisation (i.e. softwarisation) of the entire satellite hub. This means that satellite hub functional entities such as FL and RL subsystems within the baseband functions, PEP functions within satellite GWs (e.g. TCP acceleration, caching, compression) as well as control and management subsystems (e.g. NCC/NMC functions) could be implemented as software workloads instantiated on a cloud infrastructure using Infrastructure as-a Service and Platform as-a Service (IaaS/PaaS) paradigms. Each of these functions would be implemented in logically isolated virtualised appliances and chained together to become components of a satellite virtual hub (vHub) and eventually of an end-toend fully virtualised satellite network managed by a SVNO. Moreover, it is envisioned that the 
SVNO would be able to request the instantiation and customisation of satellite virtual hubs via customer portals, resulting in a service delivery model referred here as Satellite Network as a Service (SatNaaS). On-demand and pay-per-usage features form part of the envisioned SatNaaS.

Providing SatNaaS using cloud-based NFV processing and virtualisation of satellite network functions while integrating SDN techniques is the fundamental outcome of this transition that aims at bringing cost and efficiency benefits from the cloud computing model. The aim of this use case (illustrated in Figure 5) is to enable the creation of an environment with fully virtualised capabilities in order to instantiate, run, control, and manage the satellite network functionalities with intelligent processing by cloud server offering elastic, scalable, and ondemand functionalities. This will allow also increasing service availability and better interaction with core network functionalities while supporting edge applications and services [23].

A key added-value stemming from this approach, compared to current SVNO offerings, is the delegation of full administrative privileges to the SVNO, who is able to manage the entire virtual appliances, including the vHub, as it was dealing with its own physical network equipment. Thus the SVNO can enjoy similar administrative freedom compared to SNO in managing its physical satellite network. Another benefit for the SVNO is the capability to choose among multiple virtual appliances and combine (chain) them as desired. The fast setup time as well as the resource elasticity is also another advantages to be considered. This allows scaling up or down the resources assigned to the SVNO, depending on the traffic served and the customer density and demand. In this way, SVNOs could make the provisioning process of the services delivered to their customers fully automated. This capability paves the way towards fully programmable satellite virtual networks. In turn, NFV is needed for the virtualisation and unified management of the virtual appliances which are the components of the vHub, assuming that all VNFs will expose a common, standardscompliant interface for management. These interfaces will guarantee compatibility for accessing and efficient controlling of the virtual resources.

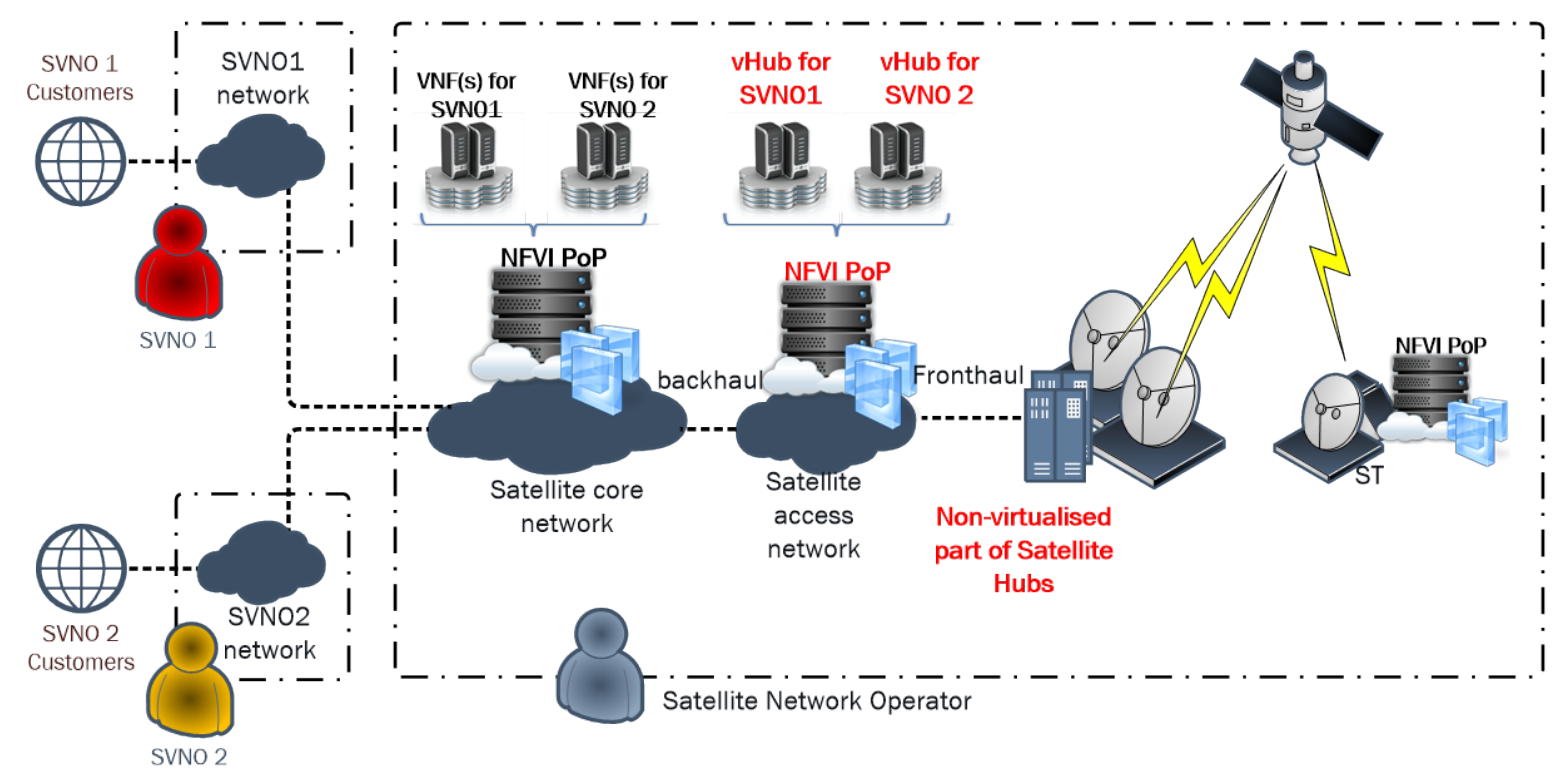

Figure 5. Illustration of Use Case "Satellite Network as a Service (SatNaaS)"

Key technical challenges stemming from this use case are: 
- Determining which satellite hub functionalities can run in software in a cloud-based environment aiming to hide the complexity of underlying technologies and network layers.

- Determining the appropriate function split between the virtualised and non-virtualised part of the satellite hub. For example, one option consists of virtualising the complete baseband unit and connecting it with the remote radio unit using a fronthauling link. This approach may bring a huge overhead on fronthaul links. Other splits are possible and deserve further investigation.

- Determining how radio resource partionining could be addressed between vHubs.

- Resiliency aspects by quantifying the potential of functionalities cloudification impact in terms of jitter, synchronization, load requirement, reliability, etc.

- Security aspects should be considered in the cloudification / softwarization process of hardware appliances.

- Determining how the SatNaaS can be seamlessly integrated with terrestrial network to support multiple path capabilities with advance options on backhauling, redundancy, reliability, traffic engineering and load balancing.

\section{4G/5G SATELLITE BACKHAULING SERVICES}

Backhauling through satellite links is one of the most compelling scenarios for the integration of the satellite component in a terrestrial mobile infrastructure. While mobile satellite backhauling solutions are a reality today, the high cost of satellite bandwidth and the technical complexities concerning the integration of satellite links into the mobile networks have largely restricted the use of satellite backhauling to remote or hard to reach locations and low traffic settings (e.g. backhauling of a base station site mainly used for the delivery of voice communications and very low data rate services). Nevertheless, technological advances in the satellite domain such as the use of HTS is changing the way that capacity is being brought to the market, reducing the price per bit and making it more attractive for Mobile Network Operators (MNOs) to backhaul mobile traffic over satellite [5]. TDMA Very Small Aperture Terminal (VSAT) solutions that support download speeds of 50- 200Mbps for backhauling 3G/LTE cells are already in the market [24][25]. Remarkably, low cost satellite backhaul together with advances in small cells create an appealing cost-efficient proposition for MNOs to explore business opportunities that otherwise would not be economically feasible [26][27]. On top of that, bringing SDN/NFV into the satellite domain is expected to further reduce the adoption barriers of the satellite component by easing the integration and use of satellite backhauling services within $4 \mathrm{G} / 5 \mathrm{G}$ mobile networks.

In addition to service extension in remote or hard to reach areas, a tighter integration of satellite backhauling services in $4 \mathrm{G} / 5 \mathrm{G}$ mobile networks can be instrumental to facilitate:

- More efficient traffic delivery to radio access network (RAN) nodes. Used in combination with a terrestrial backhaul (e.g. fibre, copper, microwave), satellite links may be used for traffic overflow, i.e. when a terrestrial backhaul link exceeds a threshold of use, close to congestion levels, the traffic excess may be routed through the satellite backhaul link, thus avoiding congestion in the terrestrial link. More challenging is the use of the satellite component for smarter traffic offloading and load balancing strategies across the terrestrial and satellite backhauling components. For example, the satellite link can be used to offload multicast/broadcast traffic addressed 
to multiple cell sites (e.g. cached content at the RAN, TV live streams for onward multicast over the RAN) in a more resource efficient manner [28].

- Increased resilience. The satellite links can be used to increase the availability and resiliency of the mobile backhaul network. Satellite service can provide additional bandwidth to divert traffic from congested areas and backup connectivity to critical cell sites so that a limited capacity in their terrestrial links can be supplemented during peak-time or even replaced in case of total / partial failure or maintenance [29].

- Fast, temporary cell deployments and moving cells. Temporary deployments require equipment that is portable and that can be rapidly installed and commissioned to provide or restore essential communications infrastructure for special events or disaster recovery [30]. Fast provisioning of satellite capacity to serve the backhauling needs of a temporary deployment is crucial. Fully integrated solutions that bundle small cells, satellite backhauling and RAN equipment (e.g. controllers), ready to be connected to a MNO core network, are being envisioned as a comprehensive approach enabling quick deployment. The deployment of small cells inside transportation facilities (i.e. small cells within buses, trains, airplanes) is also a compelling case that deserves a flexible management of the backhauling capacity, which can be partly of fully reliant on satellite communications.

Building on the enhanced flexibility (e.g. bandwidth on demand) and satellite network service customization (e.g. specific VNFs deployed per tenant, potentially also encompassing vHubs) capabilities brought by SDN/NFV into the satellite ground segment networks (as addressed within Scenario 1), the achievement of the following goals can be facilitated:

- To improve the level of control and management of satellite backhauling services by the MNO through programmable interfaces for resource management and control.

- To demonstrate the full potential of a satellite capacity component for backhauling within 4G/5G networks, not limited to remote or hard to reach locations.

- To explore the potential of extending the role of SNO not only as a provider of satellite connectivity but participating in the value chain of Mobile Edge Computing (MEC) services and cellular access capacity provisioning through the operation of neutral Radio Access Network (RAN) shared nodes (e.g. multi-tenant small cells bundled with satellite connectivity).

- To allow MNO to simplify integration and management of satellite network services to satisfy its time- and location-dependent and variable backhauling needs.

- To allow MNO to control the traffic engineering policies and mechanisms within its satellite network services (e.g. QoS control, routing policies).

- To allow MNO to reduce the deployment cost of backhaul infrastructure and improve terrestrial network resiliency.

- To allow MNO and SNO to support mechanisms for dynamic SLA (re-) negotiation of satellite network services' characteristics.

- To allow MNO to be able to dynamically and flexibly provision and configure satellite network services with specific network functions (e.g. PEP, firewalling, etc.).

- To allow MNO to be provided with mechanisms for real-time monitoring and analytics of satellite communications services.

Two use cases are addressed in the following to gain more insight into a number of key relevant features within this scenario and identify key technical challenges lying ahead. 


\subsection{Enhanced control and management of satellite backhauling capacity}

A higher level of control of the satellite network services for mobile backhauling could be achieved through the deployment of management and control interfaces between the SNO and the MNO. Figure 6 illustrates the case where the MNO contracts satellite network services from a SNO that runs a SDN/NFV-enabled satellite network. The SNO could provide the MNO with a virtual satellite network customised for mobile backhauling services, similarly to the case discussed in Section 3.2 for SVNO. Within the MNO's domain, the figure illustrates the MNO's RAN, the MNO's core network and service delivery platforms, and the MNO control and management systems (e.g. Business/Operations Support System (B/OSS) I Network Management System (NMS) and other control and management platforms that the MNO might have such as NFV orchestrators, SDN controllers, etc.). Backhauling of the RAN nodes (e.g. small cells, macro-cells, fast deployable cells, moving cells) is supported through terrestrial mobile backhaul links (e.g. backhaul based on fibre and/or terrestrial microwave links), satellite backhauling services delivered by the SNO or a combination of both.

As illustrated in Figure 6, management and control interfaces provided by the SNO could allow the MNO to flexibly control the amount and distribution of satellite backhaul capacity, directly managing some capabilities in the STs co-located with the RAN nodes as well as other functions within the satellite network. Interfaces may be related to both B/OSS for commercial and business matters [31] (e.g. dynamic SLA) and NMS for service and network level management (e.g. dynamic traffic control). Opening satellite devices via programmatic control interfaces exposed to MNOs alongside with network virtualisation capabilities (e.g. customisation of the satellite network services through the SatNaaS delivery model discussed in Section 3.3) are central enablers for the implementation of this use case [29][32][33]. Programmability may concern both the control plane, allowing MNOs to devise their own customized traffic control schemes (e.g. QoS settings control), and the data plane, allowing MNOs to devise customized packet processing algorithms (e.g. PEPs). Furthermore, the SNO could deploy a vHub in a NFV platform and delegate the full control and management of this network element to the MNO. In this way, MNOs can independently enforce their own policies on its virtual satellite network. 


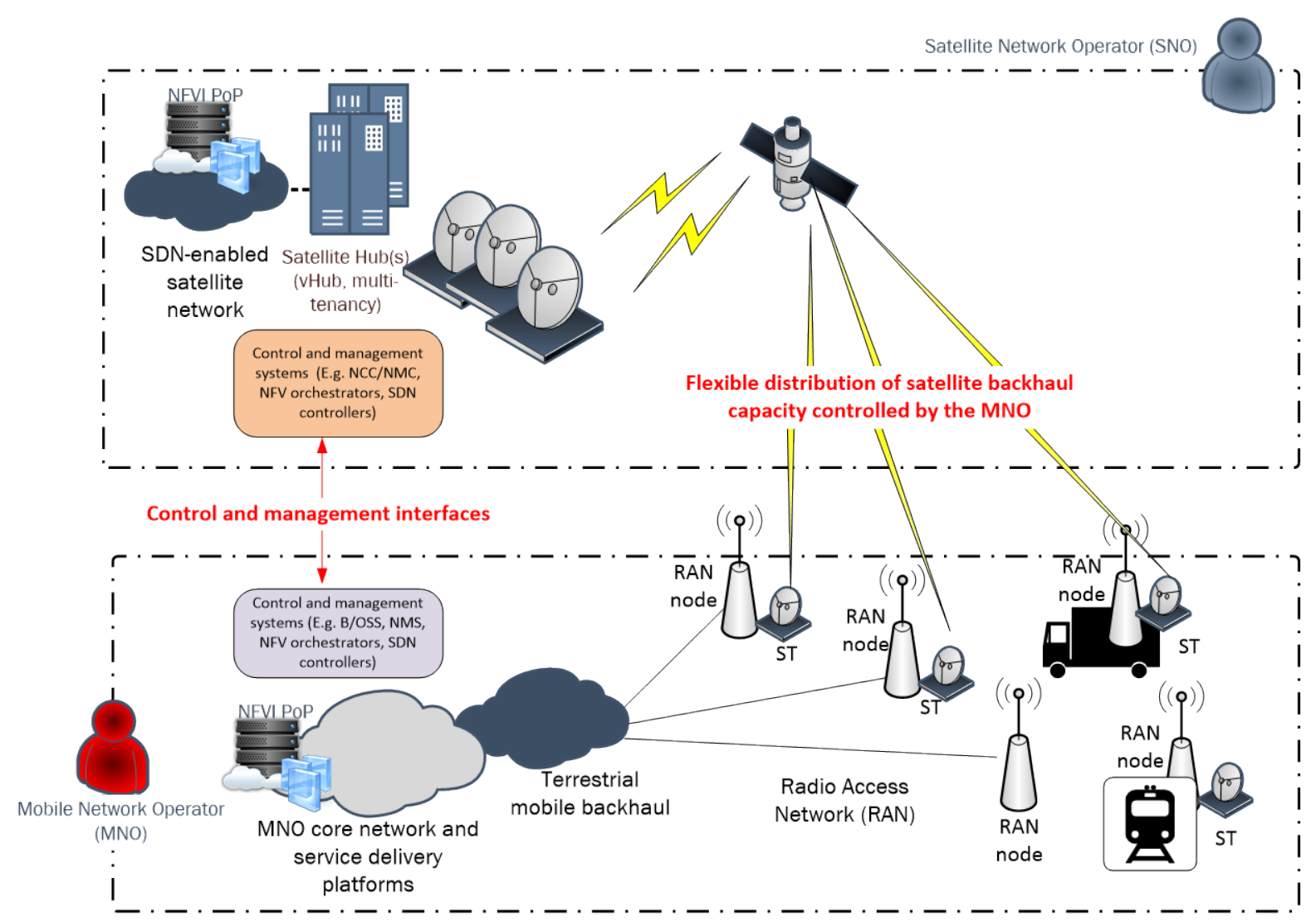

Figure 6. Illustration of Use Case "Enhanced control and management of satellite backhauling capacity"

Technical challenges arisen in this use case are:

- Definition of the proper interfaces needed to export the control and management capabilities, ensuring alignment to the emerging standards for $5 G$ networks. This is crucial for the satellite communications platforms to become an intrinsic and highly beneficial part of the $5 G$ specifications.

- Definition of the abstraction models of satellite communications services and virtual satellite networks to be used for mobile backhauling.

- Definition of a resource management framework that could allow the MNO to have end-to-end control of the mobile network resource, considering radio access capacity together with satellite and terrestrial backhauling capacity.

- Definition of a resource management framework for dynamic and flexible provisioning and configuration of multiple and diverse VNFs as part of the offered satellite backhauling service (e.g. vPEP, virtual traffic optimiser).

- Strategies for optimal capacity and traffic distribution between the terrestrial and satellite backhauling, considering multi-link transmission capabilities and specifics of traffic composition (e.g. multicast bearers / unicast bearers, QoS requirements of sessions, content caches, etc.).

- Strategies for resiliency through satellite backhauling in partially isolated RAN nodes.

- Support of dynamic SLA negotiation and bandwidth on-demand for mobile backhauling services, including scheduled bandwidth on demand to account for future foreseeable needs. 
- Flexibility in the provisioning, configuration and customisation of satellite backhauling services by the customer MNO with reduced (if any) intervention by the SNO owning the satellite ground segment platform.

\subsection{Extending satellite backhauling with edge computing services and multi-operator sharing}

In a virtualisation-enabled world, backhauling can mean much more than delivering plain connectivity and capacity. Specifically, one of the envisaged key elements of the $5 \mathrm{G}$ technological framework is the capability to deliver intelligence directly to network's edge, in the form of virtual network appliances, jointly exploiting the emerging paradigms of NFV and edge cloud computing [34]. Novel edge infrastructures promise to offer dynamic processing capabilities on-demand, optimally deployed close to the user. Following this direction, novel business cases could arise to produce added value from any kind of infrastructure or application that has the potential to be offered "as a Service".

This use case focuses on the extension of satellite backhauling services with edge computing services. Therefore, beyond allocating capacity on-demand and providing the necessary QoS per service, this use case envisions the deployment of instances of specific services of the terrestrial network, such as LTE Evolved Packet Core (EPC) components, as VNFs within the SDN/NFV-enabled satellite network. This concept is referred to as satellite edge processing, which is in line with the MEC framework under development in ETSI [34]. This concept eventually results in a totally new service mix, in which traditional backhauling is coupled with edge processing resources, offered on-demand, as-a-Service.

As illustrated in Figure 7, virtualisation capabilities for MEC could be placed on the network side of the satellite network but also collocated with the STs, which would be able to host MEC applications and other VNFs on behalf or complementing those running on the RAN nodes. In this way, the ST is essentially transformed into a virtualisation-capable remote head-end, able to serve a wide range of services. Deployment of various services and caching content at the ST can achieve significant savings in satellite capacity. Two examples for local traffic processing are:

- Service delivery platform and/or mobile packet core components (e.g. virtual IP Multimedia Subsystem [VIMS], virtual EPC, MEC applications such as location services and data caching) could be deployed within the satellite network and reduce the amount of traffic exchanged between the RAN nodes and core network of the MNO.

- In Machine-to-Machine (M2M) services, measurements from multiple sensors can be aggregated and processed locally at the ST so that only specific detected events are transmitted back over satellite. Likewise, video streams could be dynamically transcoded, its features extracted and only the features/processing results be transmitted back over satellite.

Another key capability to consider in this use case is the support of multi-tenancy in the ST. This may result in the ST partitioned into multiple "virtual ST" and serving multiple MNOs. This capability could be exploited in scenarios where the SNO intends to offer satellite access to multiple customers connected to the same STs. For example, a set of ST covering a remote village could be provided by a SNO and shared among two or more MNOs. In this 
case, RAN nodes (e.g. small cells) of the different MNOs would be attached to the same physical ST with MEC-enabled capabilities.

In addition to satellite backhauling services with edge computing services, the SNO service could be further extended to incorporate the small cell (as opposed to the small cell being provided by the MNO). This would be an evolved model where SNOs deploy by themselves wireless access capacity that is offered to MNOs in combination with the satellite mobile backhaul. Moreover, the small cells managed by the SNO could support multi-tenancy and be offered as a neutral RAN node to a number of customer MNOs. Illustrations of singleoperator settings (the small cell is not shared by MNOs) and multi-operator settings (with multiple small cells owned by multiple MNOs connected to the same ST and neutral small cells shared by the MNOs) are depicted in Figure 7.

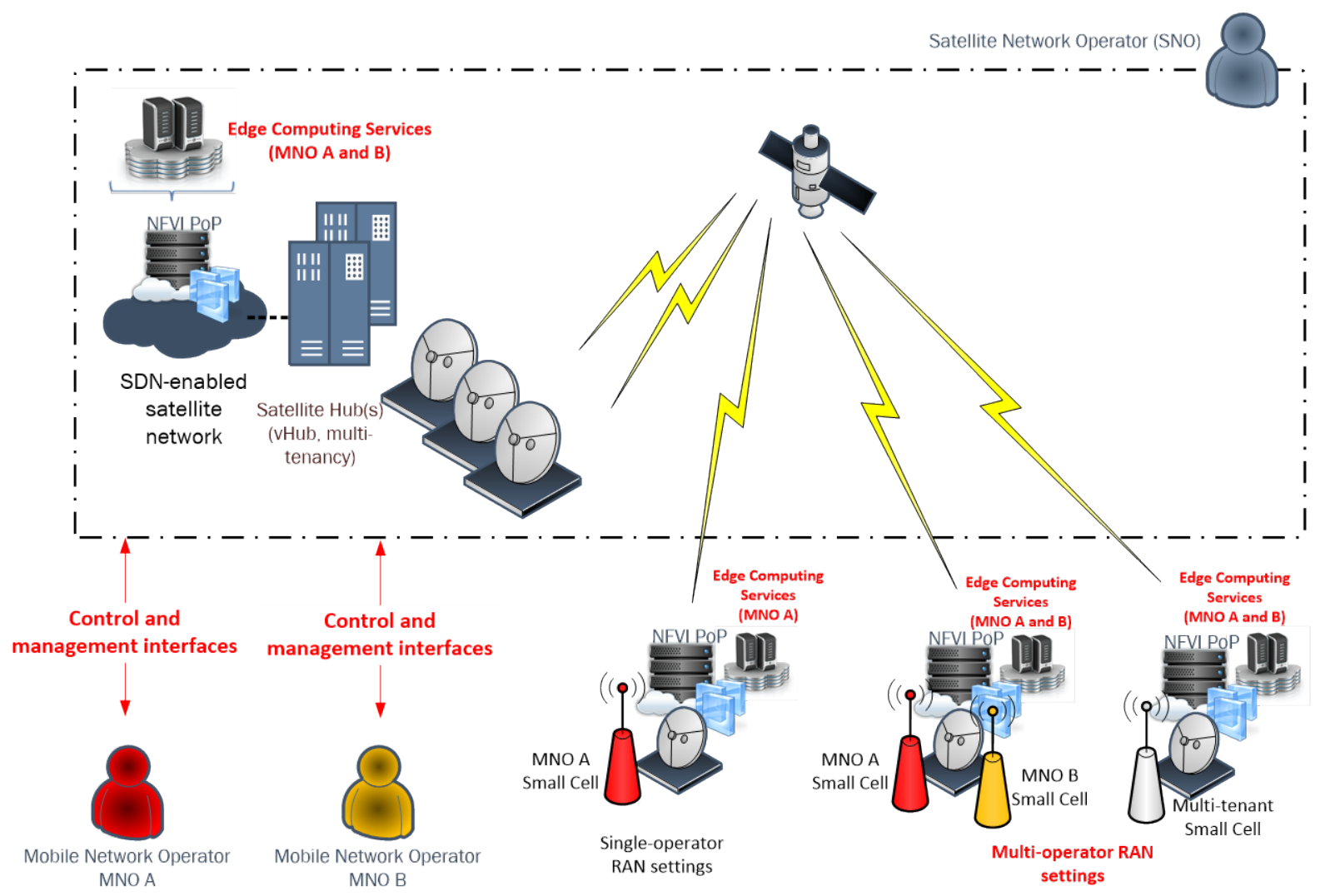

Figure 7. Illustration of Use Case "Extending satellite backhauling with edge computing services and multi-operator sharing"

Technical challenges arisen in this scenario are:

- Definition of the proper interfaces needed to export the control and management capabilities for support of edge computing services within the satellite network.

- The integration and management of VNFs and edge computing services per customer in case of multi-tenancy support.

- Sharing and multi-tenancy for multi-operator RAN settings.

- Support of agile deployment of edge computing services and VNFs that keep pace with the rapid growth and change of customer demands and context. 


\section{SATELLITE-TERRESTRIAL HYBRID ACCESS SERVICES}

Hybrid access networks are those combining a satellite component and a terrestrial component in parallel [12]. Satellite and terrestrial fixed/mobile access links properly combined together could achieve better QoS / QoE delivered to end-users as well as the plurality and the diversity of the offered services. One compelling approach to address the combination of satellite and terrestrial access networks is federation. Federation refers to the pooling of network resources from two or more domains in a way that slices of network resources distributed across the different domains can be created and used as one logical domain enabling easier control of the resources [36]. Federation of network resources in heterogeneous and multi-domain scenarios is currently being addressed in several EU research projects (e.g. FELIX [37], FI-PPP XIFI [38], and FP7-NOVI [39]). The expected development of federation capabilities in satellite communications seems very promising in the quest of providing additional resources, features and services to customers. However, this convergence is not currently favoured due to the technical complexity involved in each domain, the slow evolution of the satellite systems in contrast to the rapid evolution of terrestrial systems, the difficulty to find win-win business models, and the lack of techniques to facilitate this federation in a flexible way.

Reconfigurability, evolvability and programmability are three key characteristics that can facilitate the achievement of the federation challenge, while SDN and NFV are the most promising enabling technologies. By embracing SDN, the satellite network can expose a vendor-neutral, universally supported open interface, enabling unified management with terrestrial networks. Similarly, the NFV techniques simplify the provision of value-added networking services in the satellite communications systems, by expanding the terrestrial NFV management framework to satisfy the needs of the satellite domain as well.

The use cases described in the following focus on the federation and coupling between satellite and terrestrial systems for hybrid access, including either fixed and/or mobile networks. This is in line with the 5G vision, which encompasses federation of heterogeneous access networks in a transparent manner [40]. In terms of impacted features, federation and coupling may address various topics, such as forwarding, routing and switching, resource allocation (L2) and network management.

Federation, together with the SDN/NFV capabilities within satellite networks described in Scenario 1, is expected to facilitate the achievement of the following goals:

- Simplify the integration and management of network service functions for federated satellite and terrestrial network domains.

- It is created a new market, where the federation from a business perspective is supported by a third party e.g. a broker, which offers added value federated network services supported by the underlying federated networks.

- Isolate the required federated network service from other SVNOs and SNOs and guarantee individual managed resources and predictable performance. With the term federated network service, we consider the network services which are composed by VNFs coming from the federated network domains. Therefore the federated network service composition and provision would not be possible without successful federation. 
- The SVNOs and SNOs are members of the federation by providing and allocating part of their resources to the delegation, while the rest available resources are individually exploited by them for covering their own (non-federated) needs.

- Allowing monitoring and control of individual resources per federated network service.

- Flexibility to support and sustain new federated services derived from user (e.g. SP) demands.

- Support agile deployment of new federated services that keep pace with the rapid growth and change of user (e.g. SP) demands.

\subsection{SDN-based flexible federation of satellite and terrestrial networks}

The aim of the SDN-based flexible federation of satellite and terrestrial networks, illustrated in Figure 8 , is to advance the typical satellite broadband access network with SDN capabilities in order to achieve a vendor-neutral, universally supported interface, enabling unified management with the terrestrial networks.

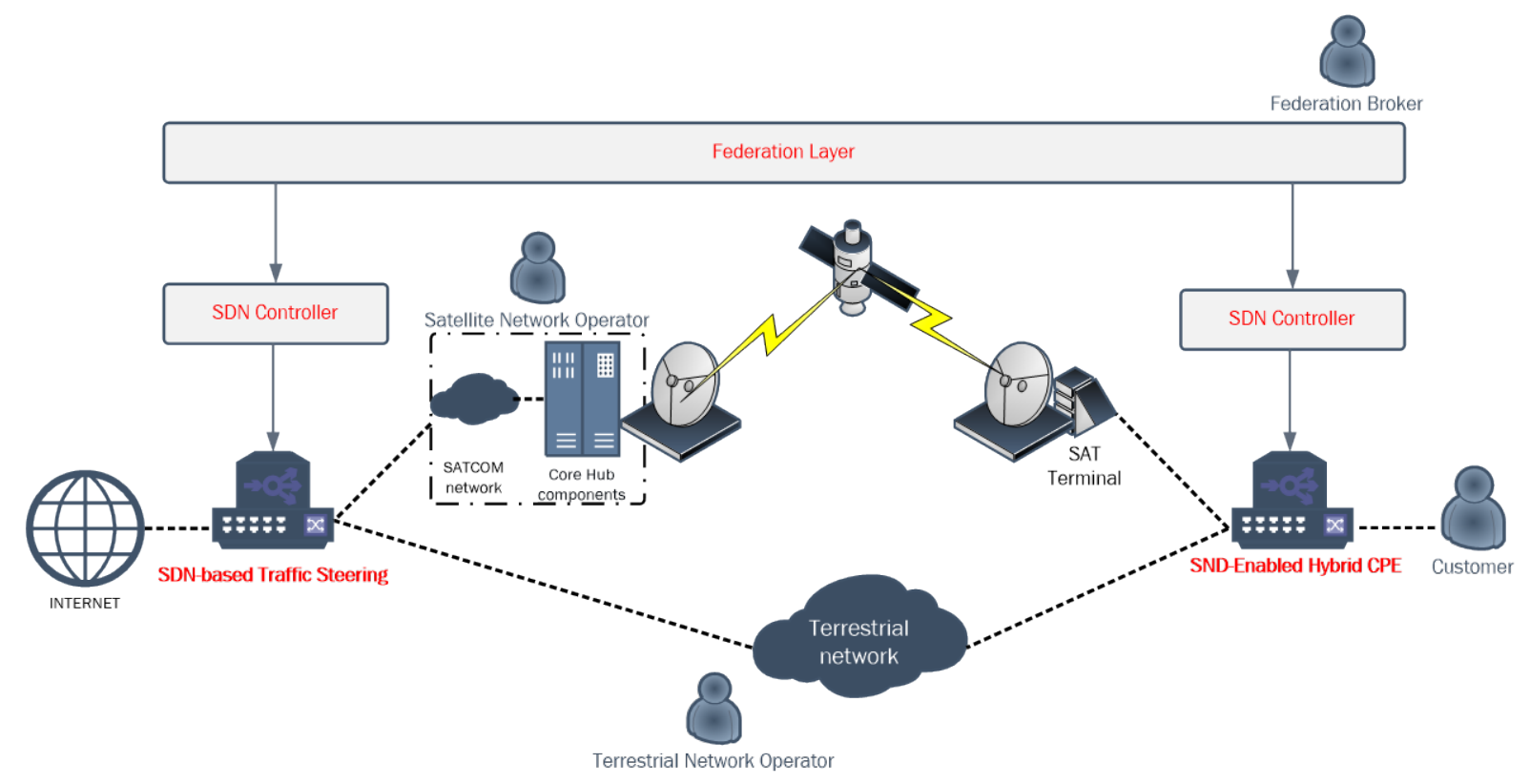

Figure 8. Illustration of Use Case "SDN-based flexible federation of satellite and terrestrial networks"

Enabling dynamic and flexible traffic steering and forwarding by SDN techniques between satellite and terrestrial access network when needed according to the best reception or the least utilized network or other conditions, leads to higher application performance and business efficiency. The SDN-based flexible federation of satellite and terrestrial networks requires that traffic control, inspection and prioritization are present before the satellite as well as the network domain. Although these operations can be achieved via traditional networking technologies (e.g. MPLS), their implementation via SDN offers much more flexibility and agility. Among the advances of applying flexible traffic steering and switching, supported by an SDN architecture with a programmatic northbound API are the centralized and granular control over the federated networking infrastructure, enabling customers to experience dynamic changes to the access network through which the requested service is delivered, either immediately depending on specific triggering conditions or scheduled in the 
future. Other advances include that the SDN controller can leverage per-flow management to cost-effectively provide guaranteed performance on a per-connection or flow basis to meet SLA requirements. Thus flow-based traffic steering can be performed, differentiating the achieved QoS of each flow due to optimal selection of the access network.

From a business perspective, this simple form of SDN-based federation between the terrestrial and the satellite network may have a significant impact in terms of high network availability and resilience. Being able to maintain the performance of critical communication systems (e.g. emergency communications systems) under various conditions by applying SDN-based seamless transition to an alternative/optimal access network (either satellite or terrestrial) in case that the current access network fails is central.

Key technical challenges stemming from this use case are:

- How to efficiently provide traffic steering/switching, achieving seamless service provision from the end-user perspective over the federated ecosystem.

- How to perform fast and dynamic traffic steering to meet the demand based on the most appropriate SDN-compatible traffic monitoring solution.

- How to define appropriate SDN rules for supporting traffic steering through multidomains and cloud-computing network domains.

\subsection{Media distribution over federated SDN/NFV-enabled terrestrial and satellite network}

The aim of this use case is to demonstrate the advances created by the federation of SDN and NFV-enabled satellite and terrestrial domains for optimized content distribution. The opportunities that are created for the hybrid distribution of digital media over an SDN/NFVenabled federated ecosystem, goes beyond the typical combination of satellite broadcasting TV with IP content (such as Hybrid Broadcast Broadband TV or second screen applications), which is achieved even today with legacy techniques. This use case will focus more on the problems that the satellite network will face by its integration with the other terrestrial networks in a dynamic and flexible manner as part of the overall $5 \mathrm{G}$ ecosystem and how SDN and NFV techniques can facilitate towards alleviating the satellite from these issues in order to maintain the QoS of the delivered service.

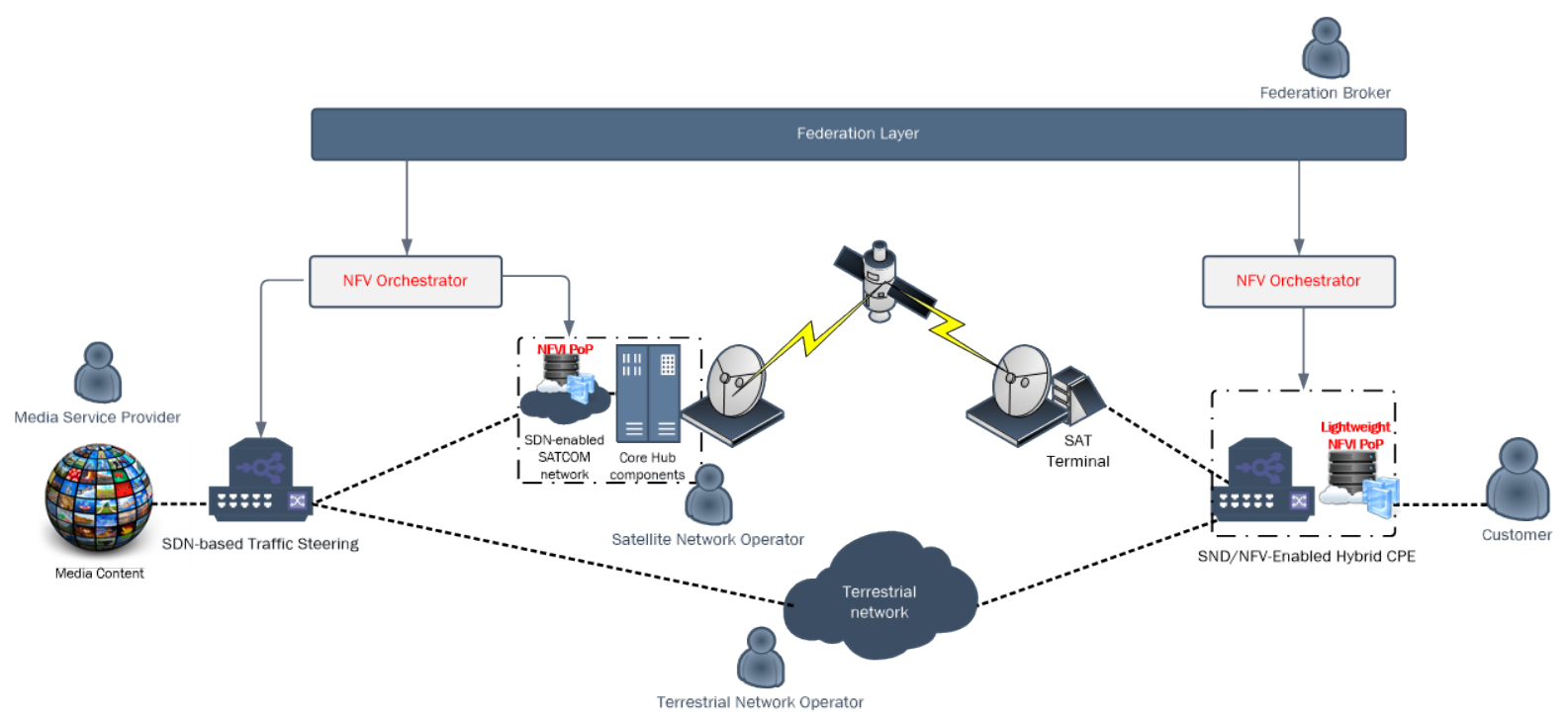


The use of SDN brings benefits both to network control and network monitoring. In terms of network control, once the network is partitioned into network slices, these slices can be offered in QoS-enabled dedicated partitions to multiple Media Service Providers (MSPs). Furthermore, on top of this, the slices can be programmable, allowing the MSP to develop an arbitrary SDN application to manipulate/divert the media streams across multiple paths as desired. This is a significant added-value compared to the current static, non-programmable federation, where the MSP just reserves the requested capacity, without any programmable or control capabilities.

The use of NFV allows either, at the MSP side or at the customer side, to deploy and instantiate dynamically VNFs that will facilitate the provision of the requested media service while aiming to maintain the appropriate QoE. For example in case of traffic congestion, while the SDN may apply appropriate traffic steering policies and route the service flow through the less utilised network of the federation, the VNF approach will deal with the congestion with the instantiation of a transcoder as a function at the MSP side, which will adapt the content dynamically in order to facilitate its provision. Therefore, the combination of both SDN and NFV techniques provides a plethora of choices for applying traffic steering of the media service or performing dynamically adaptation or other combined actions depending on the problem and the way of resolving it.

In this use case, satellite and/or terrestrial network operator(s) are assumed to count with virtualisation mechanisms to partition their networks and lease slices to MSPs. A new role for federation brokering is also envisioned as an intermediate player between network operators and MSPs. In this way, MSPs would lease the slices through the federation broker in order to offer media services to end-users, who are equipped with either single- or hybrid-access terminals, i.e. attached simultaneously to both satellite and terrestrial access network.

Key technical challenges stemming from this use case are:

- How to efficiently integrate and manage VNFs per MSP in a multiple tenants environment.

- How to isolate the required MSP capacity from other MSP with appropriate SDN policy techniques.

- How to provide resources adjustment when MSP expand or shrink their resources, providing monitoring and control of individual MSP resources, and finally providing flexible and efficient virtual to physical resources mapping.

- How to deploy and instantiate media-related VNFs without interrupting the service.

- How to enable the coordination logic for federation and combining multiple actions in terms of SDN and NFV decisions without interrupting the media delivery.

- How to support of agile deployment of new network services and VNFs that keep pace with the rapid growth and change of MSP's demands.

\subsection{Customer functions virtualisation over federated terrestrial and satellite network}

The aim of this use case is to demonstrate the VNF-as-a-Service (VNFaaS) paradigm and assumes the dynamic offering by the SVNO/SNO of virtual network appliances to customers in the form of VNFs (e.g. load balancers, firewalls, traffic filters, home gateway functionalities, 
media storage and processing etc.). The provision in a multi-tenant way (i.e. per customer) of such capabilities is currently very costly, making practically the network functionalities at the satellite gateway to apply to the entire traffic and of course not being manageable by the customer.

According to their nature, these VNFs can be instantiated either at NFVI PoPs within the satellite network infrastructure or at VNF-enabled STs or at other parts of the terrestrial segment (considering the federated terrestrial and satellite network of this use case). For example, firewalling should be conducted at the satellite gateway to avoid transmitting over satellite traffic which will be eventually blocked at the terminal.

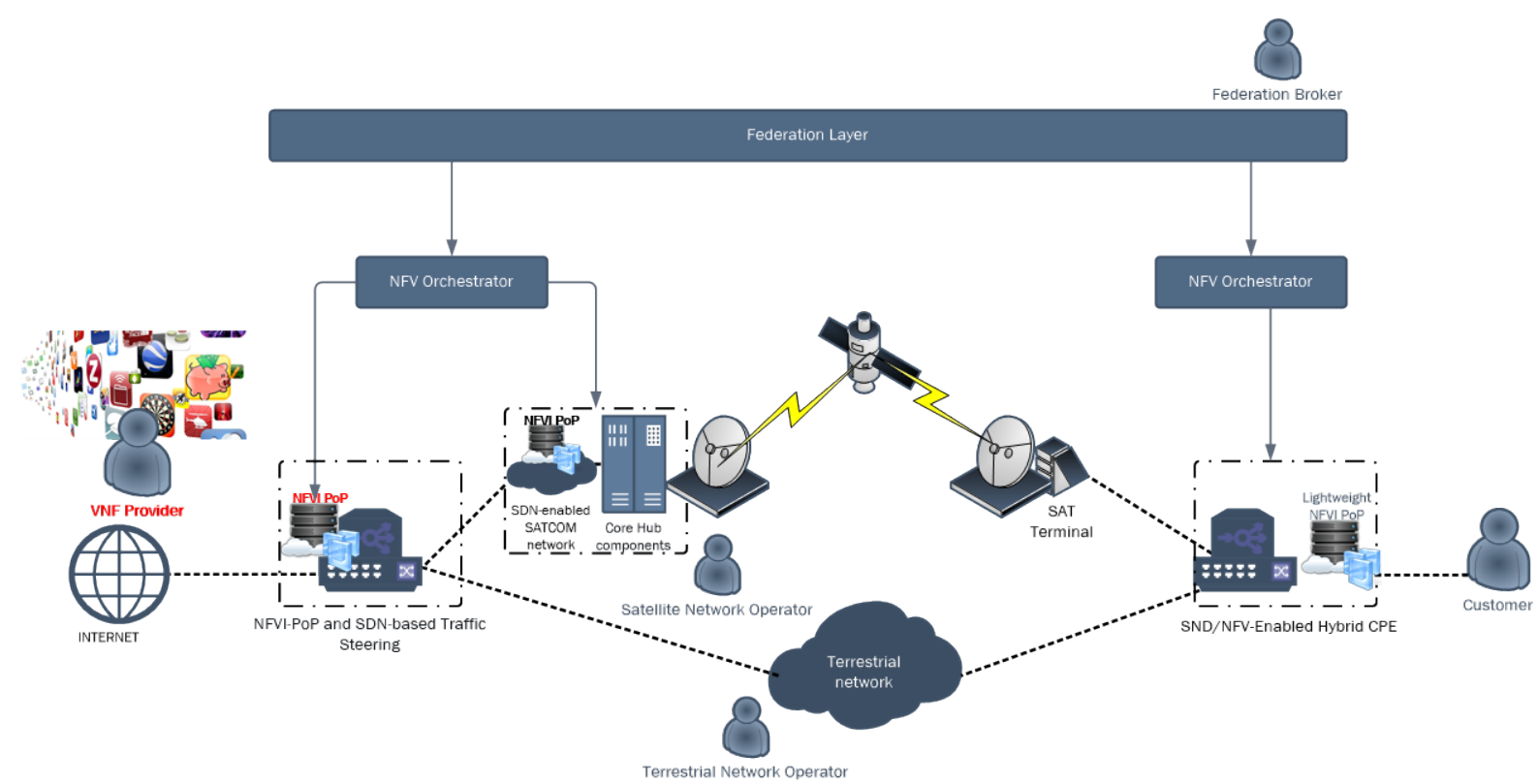

Figure 10. Illustration of Use Case "Customer functions virtualisation over federated terrestrial and satellite network"

The NFV platform needs to accommodate interactions with the customers, allowing them to select, deploy, manage and monitor VNFs. An NFV service catalogue is essential to be offered at the federation level in order to allow customers to customize the services according to their needs. One possibility is that the SNO also undertakes the role of the NFV service provider and offers VNFaaS as added-value services along with satellite connectivity to customers. Another possibility is that the VNF Providers (developers) play a more active role, advertising and dynamically pricing their services which are published in a catalogue. The customers may select the services that best suit their needs. In some business models, the VNF Providers may receive direct profit from the customers, either indirectly as a share of the satellite communications service fee or directly, as a license fee for using the VNF.

Key technical challenges stemming from this use case are:

- The service composition and service chaining of various VNFs performed by the Federation Layer.

- The traffic steering mechanism needed to be applied by the Federation Layer in order to support a multi-domain network service provision.

- The VNFaaS placement logic for selecting the appropriate NFVI PoP depending on the type of the service and the type of action, as well as the special requirements of each VNF. 
- The coordination logic for federation and combining multiple actions in terms of SDN and NFV decisions to support the instantiation and deployment of VNFaaS.

- The orchestration of the VNFaaS lifecycle through the appropriate monitoring and adaptation framework reassuring flawless service delivery.

\section{CONCLUSIONS}

The role that satellite communications can play in the forthcoming $5 \mathrm{G}$ ecosystem is being revisited. In particular, the adoption of SDN and NFV technologies into the satellite domain is seen as a key facilitator to make satellite communications to become a constituent part well integrated within an anticipated multi-layer/heterogeneous $5 \mathrm{G}$ network architecture.

Through the description of relevant scenarios and use cases, this paper has investigated how SDN and NFV technologies can enhance the interoperability of the satellite networks and the deployment of services across hybrid satellite-terrestrial configuration variants. Three main scenarios have been identified and analysed, focusing on the improvement of satellite network infrastructures (Scenario 1) as well as on the improvement of combined terrestrialsatellite offerings such as satellite backhauling services to $4 \mathrm{G} / 5 \mathrm{G}$ networks (Scenario 2 ) and satellite-terrestrial hybrid access services (Scenario 3).

Multiple opportunities and benefits for the different actors (SNO, SVNO, MNO, MSP, etc.) have been identified for each scenario, along with new business opportunities (e.g. bundling of backhauling with MEC and neutral RAN nodes, federation of satellite-terrestrial networks, VNF providers for satellite communicates, etc.). However, it has been shown that the full realisation of the described scenarios and use cases still raises multiple technical challenges that have to be researched.

Based on the characterisation of the scenarios and use cases provided in this paper, architectural and related resource management solutions for SDN/NFV-enabled satelliteterrestrial networks are currently being investigated by European Union H2020 VITAL research project [11].

\section{LIST OF ACRONYMS}

API: Application Programming Interface

ASP: Application Service Provider

CPE: Customer Premises Equipment

EPC: Evolved Packet Core

FL: Forwarding Link

GW: Gateway

HTS: High Throughput Satellite

IMS: IP Multimedia Subsystem

ISP: Internet Service Provider

MEC: Mobile Edge Computing

MNO: Mobile Network Operator

MPLS: Multi-Protocol Label Switching

MSP: Media Service Provider

NAP: Network Access Provider

NCC: Network Control Centre

NFV: Network Function Virtualisation

NFVI: NVF Infrastructure

NMC: Network Management Centre

NMS: Network Management System 
NSP: Network Service Provider

Ln: Layer $n(n=0 . .7)$

ODU: Outdoor Unit

PEP: Performance Enhancing Proxy

PoP: Point of Presence

QoE: Quality of Experience

QoS: Quality of Service

RAN: Radio Access Node

RL: Returning Link

RRM: Radio Resource Management

SatNaaS: Satellite Network -as-a-Service

SDN: Software Defined Networking

SLA: Service Level Agreement

SNO: Satellite Network Operator

SO: Satellite Operator

SP: Service Provider

ST: Satellite Terminal

SVNO: Satellite Virtual Network Operator

vHub: Virtual Hub

VNF: Virtual Network Function

VNFaaS: VNF-as-a-Service

VPN: Virtual Private Network

VSAT: Very Small Aperture Terminal

\section{ACKNOWLEDGEMENT}

Research leading to these results has received funding from the European Union's H2O2O Research and Innovation Programme (H2020-ICT-2014-1) under the Grant Agreement H2020-ICT-644843.

\section{REFERENCES}

[1] ETSI TR 103124 V1.1.1, "Satellite Earth Stations and Systems (SES); Combined Satellite and Terrestrial Networks scenarios", July 2013

[2] Evans, B.; Werner, M.; Lutz, E.; Bousquet, M.; Corazza, G.E.; Maral, G.; Rumeau, R., "Integration of satellite and terrestrial systems in future multimedia communications," Wireless Communications, IEEE , vol.12, no.5, pp.72,80, Oct. 2005

[3] Cisco EPC SatRAN Satellite Radio Access Network

[4] The Integral SatCom Initiative (ISI), "SatCom role in 5G networks", Net!Works Event 2013 Implementing H2020, Brussels, 29.10 .13

[5] Sacchi, C.; Bhasin, K.; Kadowaki, N.; Vong, F., "Toward the "space 2.0" Era [Guest Editorial]," Communications Magazine, IEEE , vol.53, no.3, pp.16,17, March 2015

[6] Fenech, H.; Amos, S.; Tomatis, A.; Soumpholphakdy, V., "High throughput satellite systems: An analytical approach," in Aerospace and Electronic Systems, IEEE Transactions on , vol.51, no.1, pp.192-202, January 2015

[7] Farooq Khan, "Mobile Internet from the Heavens", arXiv.org Computer Science, arXiv:1508.02383, 9 Aug 2015

[8] ARTES programme, "ESA announces dedicated support for the development of megaconstellations", Last updated July 2015. Available online at https://artes.esa.int/news/esa-announces-dedicated-support-developmentmegaconstellations,

[9] ARTES programme, "Flexible payloads key for satcom industry", Last updated July 2015. Available online at https://artes.esa.int/news/flexible-payloads-key-satcom-industry

[10] R. Ferrús, O. Sallent, T. Rasheed, A. Morelli, H. Koumaras, G. Agapiou, C. Boustie, P. 
Gélard, R. Mestari, H. Makis, T. Masson, "Enhancing Satellite \& Terrestrial Networks Integration through NFVISDN technologies", IEEE COMSOC MMTC E-Letter http://www.comsoc.org/ mmc 17/55 Vol.10, No.4, July 2015

[11] H2020 VITAL research project website at http://www.ict-vital.eu/

[12] ETSI TR 103272 V1.1.1, Satellite Earth Stations and Systems (SES); "Hybrid FSS satellite/terrestrial network architecture for high speed broadband access", March 2015

[13] Gerard Maral, Michel Bousquet, Zhili Sun (Contributing Editor), "Satellite Communications Systems: Systems, Techniques and Technology, 5th Edition, ISBN: 978-0-470-71458-4, December 2009

[14] ETSI TS 101 545-1 V1.2.1 (2014-04), "Digital Video Broadcasting (DVB); Second Generation DVB Interactive Satellite System (DVB-RCS2); Part 1: Overview and System Level specification", April 2014. Available online at http://www.etsi.org/deliver/etsi ts/101500 101599/10154501/01.02.01 60/ts 10154501v 010201p.pdf

[15] Broadband Forum, Technical Report 101 on "Migration to Ethernet-Based Broadband Aggregation", July 2011. Available at https://www.broadbandforum.org/technical/download/TR-101 Issue-2.pdf

[16] ETSI TR 101984 V1.2.1, Satellite Earth Stations and Systems (SES); "Broadband Satellite Multimedia (BSM); Services and architectures", December 2012

[17] Dave Bettinger, "Maximizing the HTS Opportunity: Leveraging New Satellite Architectures and Business Models to Grow", Asia Pacific Satellite communications council (APSCC), APSCC 2013 Q3 newsletter, ISSN 1226-8844; URL: http://www.apscc.or.kr/upload/pdf/Q3\%202013.pdf

[18] Kreutz, D.; Ramos, F.M.V.; Esteves Verissimo, P.; Esteve Rothenberg, C.; Azodolmolky, S.; Uhlig, S., "Software-Defined Networking: A Comprehensive Survey," in Proceedings of the IEEE, vol.103, no.1, pp.14-76, Jan. 2015

[19] ETSI GS NFV 001 V1.1.1, "Network Function Virtualisation; Use Cases", October 2013

[20] "Virtual Network. Real Dollars", Open Networking Forum (ONF) website, March 2013. Available online

at https://www.opennetworking.org/?p=488\&option=com_wordpress\&ltemid=450

[21] Robert Kenny, Tom Broughton, "Domestic demand for bandwidth: An approach to forecasting requirements for the period 2013-2023", November 2013. Available online at http://www.broadbanduk.org/wp-content/uploads/2013/11/BSG-Domestic-demand-forbandwidth.pdf

[22] P. Barsocchi et al., "Radio resource management across multiple protocol layers in satellite networks: a tutorial overview", International Journal of Satellite Communications and Networking, 23.5 (2005): 265-305.

[23] ETSI ISG MEC, "Mobile-Edge Computing - Introductory Technical White Paper, September 2014

[24] http://www.gilat.com/Cellular-Backhaul

[25] http://www.o3bnetworks.com/o3b-cell-mobile-backhaul/

[26] iDirect, "Extending 3G and 4G Coverage to Remote and Rural Areas Solving The Backhaul Conundrum", White paper

[27] "Backhaul for rural and remote small cells", Small cell forum, release five, white paper, March 2015.

[28] Breiling M., Zia W., Sanchez de la Fuente Y., Mignone V., Milanesio D., Fan Y., Guta M., "LTE Backhauling Over MEO-Satellite", Advanced Satellite Multimedia Systems Conference, IEEE, pp. 174 - 181, September 2014

[29] Watts S., Glenn O., "5G resilient backhaul using integrated satellite networks", Advanced Satellite Multimedia Systems Conference, 7th, IEEE. Pp. 114-119, September 2014

[30] Casoni M., Gracia C., Klapez M., Patriciello N., Amditos A., Sdongos E., "Integration of Satellite and LTE for Disaster Recovery", IEEE Communications Magazine, pp. 47-53. March 2015

[31] iDirect, "Fighting Commoditization by creating value with your NMS", white paper, 
February 2015

[32] Bertaux L., Medjiah S., Berthou P., Abdellatif S., Hakiri A., Gelard P., "Software Defined Networking and Virtualisation for Broadband Satellite Networks". IEEE Communications Magazine, pp. 54-60, March 2015

[33] Kapovits A., Covaci S., Ververidis C., Siris V., Guta M., "Advanced Topics in Service Delivery over Satellite Terrestrial Networks", Advanced Satellite Multimedia Systems Conference and the 13th Signal Processing for Space Communications Workshop (ASMS/SPSC), 7th. pp. 92-98, 2014

[34] Li, Qian; Niu, Huaning; Papathanassiou, Apostolos; Wu, Geng, "Edge Cloud and Underlay Networks: Empowering 5G Cell-Less Wireless Architecture," in European Wireless 2014; 20th European Wireless Conference; Proceedings of , vol., no., pp.1-6, 14-16 May 2014

[35] Mobile-Edge Computing - Introductory Technical White Paper, September 2014. Available online at https://portal.etsi.org/Portals/0/TBpages/MEC/Docs/Mobileedge_Computing_-_Introductory_Technical_White_Paper_V1\%2018-09-14.pdf

[36] S. Wahle, T. Magedanz, "Network Domain Federation -An Architectural View on How to Federate Testbeds", White paper available online at http://www.ictfireworks.eu/fileadmin/events/FIREweek/FIRE Strategy WS/FSWS Wahle.pdf

[37] Felix project (FEderated Test-beds for Large-scale Infrastructure eXperiments). Official website at http://www.ict-felix.eu/

[38] XIFI Project. Official website at https://www.fi-xifi.eu/home.html

[39] NOVI Project (Network Innovation over Virtualized Infrastructures). Official website at http://www.fp7-novi.eu/

[40] NetWorld2020, "Public Private Partnership in Horizon 2020: Creating a Smart Ubiquitous Network for the Future Internet", November 2013. Available online at http://networld2020.eu/wpcontent/uploads/2014/05/Advanced 5G Network Infrastructure PPP in H2020 Final November 2013.pdf 